\title{
MRI Visible Drug Eluting Magnetic Microspheres for Transcatheter Intra-Arterial Delivery to Liver Tumors
}

\author{
Dong-Hyun $\mathrm{Kim}^{1 凶}$, Jeane Chen ${ }^{2}$, Reed A. Omary ${ }^{3}$, Andrew C. Larson ${ }^{4}$ \\ 1. Department of Radiology, Northwestern University, Chicago, IL 60611, USA; Robert H. Lurie Comprehensive Cancer Center, Chicago, \\ IL 60611, USA. \\ 2. Department of Radiology, Northwestern University, Chicago, IL 60611, USA. \\ 3. Department of Radiology and Radiological Sciences, Vanderbilt University Medical Center, Nashville, TN, USA. \\ 4. Department of Bioengineering, University of Illinois at Chicago, Chicago, IL, USA; Department of Electrical Engineering and Computer \\ Science, Evanston, IL, USA; International Institute of Nanotechnology (IIN), Northwestern University, Evanston, IL, USA; Robert H. \\ Lurie Comprehensive Cancer Center, Chicago, IL 60611, USA. Department of Radiology, Northwestern University, Chicago, IL 60611, \\ USA.
}

$\triangle$ Corresponding author: Department of Radiology, Northwestern University, 710 N Fairbanks Ct. Olson 8-317, Chicago, IL 60611, USA. Tel.: 1-312-503-1307; Fax: 1-312-926-5991, dhkim@northwestern.edu (Dong-Hyun Kim)

(c) 2015 Ivyspring International Publisher. Reproduction is permitted for personal, noncommercial use, provided that the article is in whole, unmodified, and properly cited. See http://ivyspring.com/terms for terms and conditions.

Received: 2014.10.15; Accepted: 2015.01.09; Published: 2015.02.07

\begin{abstract}
Magnetic resonance imaging (MRI)-visible amonafide-eluting alginate microspheres were developed for targeted arterial-infusion chemotherapy. These alginate microspheres were synthesized using a highly efficient microfluidic gelation process. The microspheres included magnetic clusters formed by USPIO nanoparticles to permit MRI and a sustained drug-release profile. The biocompatibility, MR imaging properties and amonafide release kinetics of these microspheres were investigated during in vitro studies. A xenograft rodent model was used to demonstrate the feasibility to deliver these microspheres to liver tumors using hepatic transcatheter intra-arterial infusions and potential to visualize the intra-hepatic delivery of these microspheres to both liver tumor and normal tissues with MRI immediately after infusion. This approach offer the potential for catheter-directed drug delivery to liver tumors for reduced systemic toxicity and superior therapeutic outcomes.
\end{abstract}

Key words: MRI, microspheres, liver tumors

\section{Introduction}

Chemoembolization is an effective catheter-directed technique for palliative treatment of hepatocellular carcinoma (HCC) involving selective infusion of both chemotherapy drugs mixed with lipiodol and embolic materials into the hepatic artery feeding the tumor. Liver tumors derive their blood supply mainly from the hepatic artery, whereas the portal venous system serves as the main blood supply to normal liver tissues.[1] Local delivery affords significant reductions in systemic toxicity. Transcatheter arterial chemoembolization (TACE) has been used as a palliative treatment for patients with unresectable HCC. However, TACE offers only modest improve- ments in long-term patient survival over best supportive care. $[2,3]$ The efficacy of conventional TACE approaches may be limited by the lack of effective drugs and/or drug carriers. [4, 5]

To overcome the limitations of prior generation TACE techniques, drug-eluting beads (DEB) were recently developed for transcatheter delivery to HCC. This new type of TACE procedure involves infusion of DEBs into tumor-feeding arteries thus permitting a somewhat more sustained release of drugs into the tumor blood supply. However, despite encouraging safety profiles, these DEBs (typically used to deliver doxorubicine) have yet to demonstrate significant 
improvements in treatment outcomes compared to conventional TACE methods. A combination of modern multi-functional drug carriers with high efficiency drugs may be critical to improve therapeutic outcomes following these catheter-directed procedures. High doses of drugs can be loaded onto drug carriers in a reproducible manner for controlled elution over an extended period of time (current DEBs microspheres offer only a rapid burst release profile).[6, 7]

Amonafide (AMN) is an anti-neoplastic agent that acts as a DNA intercalator; it has demonstrated significant activity against various types of cancer. However, the drug has a 5-position amine that is acetylated to form a toxic metabolite (N-acetyl metabolite) in humans (Fig. 1a), causing serious adverse effects and complicating dosing. The toxic metabolite of amonafide is produced by $\mathrm{N}$-acetyl transferase 2 (NAT2)[8], which is a polymorphic enzyme causing differential acetylation activity among individuals[9] (Fig. 1a). The unacceptable side effects have limited patient tolerance, increased adverse effects and complicated dosing of these drugs. In HCC, systemic chemotherapy may not be an available option because the patients are particularly vulnerable to treatment-related adverse effects. Therefore, a targeted delivery system for AMN should be highly valuable to potentially ellicit potent tumor responses while avoiding the aforementioned toxicities.

Many different biocompatible polymers such as chitosan[10], PLGA[11], gelatin[12], and starch[13] have been studied for use as drug carriers; one particularly promising biopolymer is alginate polysaccharide. Alginate polymers have been used widely as a material for production of drug carriers due to their biocompatibility and biodegradablilty. Alginate is a linear anionic polysaccharide composed of two uronic acid monomers: the (1-4)-linked $\beta$-L-guluronic ( $\mathrm{G}$ block) and $a-D$-mannuronic acids (M block).[14] These monomers are connected by glycosidic bonds and appear as either homopolymeric blocks ( $\mathrm{G}$ or $\mathrm{M}$ blocks) or alternative blocks (MG blocks) in alginate. Alginate crosslinked matrix can be formed quickly via contact between $G$ blocks with divalent cations.[15] The rapid gelation process under mild conditions readily permits encapsulation of functional agents such as chemotherapeutic drugs and imaging probes.

To date, the production of the alginate microspheres has been accomplished by using electrostatic dripping, extrusion, dropping, emulsification, spray-drying and coacervation methods.[16] However, these synthetic processes have well-known drawbacks such as harsh operating conditions, cytotoxic crosslinking agents, limited size ranges and most importantly non-uniform particle or microsphere sizes with broad overall distributions. Non-uniform microsphere sizes can be problematic for trans-arterial catheter-directed delivery procedures. Rigorous control of microsphere size is particularly important in these settings because the microsphere size ultimately determines the biodistribution distal to the tip of the infusion catheter (if too small, the microspheres may pass through the tumor vascularture and onward to the lungs or if too large, the microspheres may not transit sufficiently deep into the targeted tumor vasular tree).

Microfluidic techniques should permit highly controlled formation of Ca-alginate drug carriers in the size range of one to hundreds of micrometers (considered potentially ideal for targeted transcatheter deliver procedures to HCC). These microfluidic approaches should importantly permit synthesis of microspheres encapsulating not only a therapeutic moiety but also potential imaging probes for non-invasive imaging following catheter-directed infusion. In vivo visualization of microsphere delivery to the targeted liver tumors should permit patient-specific adjustments to individual treatment protocols (additional infusions to achieve an optimal tumor dose or potentially early prediciton of outcomes to adoption of alternative treatment strategies as needed).

In this study, magnetic resonance imaging (MRI)-visible amonafide-eluting alginate microspheres were developed for targeted arterial-infusion chemotherapy.These alginate microspheres were synthesized using a highly efficient microfluidic gelation process. Magnetic clusters formed by USPIO nanoparticles were utilized for MR imaging and drug-release control agents. The biocompatibility, MR imaging properties and AMN release kinetics of these microspheres were investigated during in vitro studies. A xenograft rodent model was used to demonstrate a) feasibility to deliver these microspheres to liver tumors using hepatic transcatheter intra-arterial infusions and b) potential to visualize the intra-hepatic delivery of these microspheres to both liver tumor and normal tissues with MRI immediately after infusion.

\section{Materials and Methods}

\section{Chemicals}

$\mathrm{FeCl}_{3}$, polyacrylic acid (PAA), sodium hydroxide, diethylene glycol (DEG), sodium alginate, calcium chloride, span 80 and $\mathrm{n}^{\prime}$-hexadecane were obtained from Sigma-Aldrich (USA). Amonafide (AMN) was purchased from Selleckchem. Co. (USA). 
3-(4,5-dimethylthiazol-2-yl)-2,5-diphenyltetrazolium bromide (MTT, Sigma, USA) and dimethylsulfoxide (DMSO, Amresco, USA) were used for MTT assays.

\section{Microfluidic Fabrication of MRI Visible Drug Eluting Microspheres}

Magnetic clusters were synthesized using a high temperature hydrolysis reaction.[17, 18] Briefly, a $\mathrm{NaOH} / \mathrm{DEG}$ stock solution was prepared by dissolving $2 \mathrm{~g}$ of $\mathrm{NaOH}$ in diethylene glycol (DEG) (20 $\mathrm{ml}$ ). The solution was heated to $120^{\circ} \mathrm{C}$ for $30 \mathrm{mins}$ under nitrogen, cooled, and kept at $70^{\circ} \mathrm{C}$. A mixture of $\mathrm{FeCl}_{3}(0.4 \mathrm{mmol})$, polyacrylic acid (PAA) $(4 \mathrm{mmol})$, and DEG $(17 \mathrm{ml})$ was heated to $220{ }^{\circ} \mathrm{C}$ in a nitrogen atmosphere for $30 \mathrm{~min}$ with vigorous stirring to form a transparent solution. $\mathrm{NaOH} / \mathrm{DEG}$ stock solution (2 $\mathrm{ml}$ ) was injected into the hot mixture. The resulting mixture was further heated for $1 \mathrm{~h}$ to yield $\sim 42 \mathrm{~nm}$ magnetic clusters composed of $\sim 6 \mathrm{~nm}$ iron oxide nanoparticles. The final products were washed with a mixture of Milli-Q water and ethanol 3 times and re-dispersed in Milli-Q water. Amonafide (AMN;10 $\mathrm{mM}$ ) was loaded on the magnetic clusters by mixing in ethanol/ $\mathrm{H}_{2} \mathrm{O}(1: 1, \mathrm{v} / \mathrm{v})$ mixture, centrifuge and drying adsorption processes. Microfluidic chips with a channel dimension of $130 \mathrm{um}$ in depth and width were fabricated by conventional soft lithography techniques. A two-step droplet gelation process was adopted to produce magnetic alginate microspheres. An oil, used as the continuous phase, was composed of $n^{\prime}$-hexadecane and span $80(2 \% \mathrm{w} / \mathrm{w})$. An aqueous mixture solution of sodium alginate $(2 \% \mathrm{w} / \mathrm{w})$ and AMN-magnetic clusters (20 wt $\%$ of alginate amount) was prepared for the dispersed phase. All flow streams were individually driven using independent syringe pumps (New Era NE-1000, NY, USA) to maintain a constant flow rate. The inlet ports were connected to the syringes $(20 \mathrm{ml})$ using Tygon tubing (.02 inch I.D). A microscope equipped with a variable zoom system was used for observation (Olympus, Japan). The generated microspheres by shearing dispersed phase were collected in a calcium chloride $(50 \mathrm{mM} ; 20 \mathrm{ml})$ reservoir. The resulting magnetic microspheres were separated from the oil solution with a centrifuge and magnetic separation. These were subsequently washed 3 times with $30 \mathrm{~mL}$ of Milli-Q water.

\section{Characterization of Microsphere Size and Magnetic Properties}

The morphologies and magnetic properties of the synthesized microspheres were characterized with an optical microscope, scanning electronic microscope, confocal laser-scanning microscope and superconducting quantum interface device (SQUID).
300 microspheres within these optical images were randomly selected to estimate the average size and size distribution. Images were captured using a video camera (QColor5, Olympus, Japan) connected to a light microscope (CKX41, Olympus, Japan); these images were analyzed with the aid of image processing software (Image J). Magnified morphologies of the magnetic microspheres were observed using both a confocal laser scanning microscope (Zeiss LSM 510 META laser scanning confocal, Germany) and Scanning Electron Microscope (JEOL, JSM-5200, Japan).

\section{Amonafide Drug Loading Efficiency and Re- lease Studies}

Drug loading efficiency was measured as the ratio between the initially supplied $w t \%$ amonafide dose during synthesis and amount determined to finally be loaded in the microspheres. The loaded AMN amounts were measured by dissolving the AMN-loaded magnetic microspheres in a $50 \mathrm{mM}$ ethylenediaminetetraacetic acid (EDTA) solution with sonication for 10 mins. Drug elution studies for each set of microspheres was performed to compare AMN release kinetics for microspheres incorporating increasing amounts of magnetic clusters ( 0 and $20 \mathrm{wt} \%$ ) at $37{ }^{\circ} \mathrm{C}$. The aqueous sample solution $(30 \mathrm{mg} / \mathrm{ml} ; 1$ $\mathrm{ml}$ ) was placed in a membrane bag (Spectra/Por MWCO 10,000, Spectrum, Los Angeles, CA, USA) and then immersed in $40 \mathrm{ml}$ of PBS (Phosphate Buffer Solution, $\mathrm{pH}$ 7.2) solution. The temperature of the medium was maintained at $37^{\circ} \mathrm{C}$ using a water bath. At specific time intervals, PBS $(1 \mathrm{ml})$ medium was extracted and replaced with fresh medium. The extracted PBS was diluted with ethanol $(1: 1 \mathrm{v} / \mathrm{v})$ to measure AMN concentrations. Concentrations of released AMN were determined using fluorescent spectroscopy (SpectraMax M5, Molecular Devices, CA, USA). These measurements were performed three times and averaged to determine percentages of cumulative drug release amounts over time. The AMN loaded magnetic microspheres were also imaged using confocal fluorescent microscopy at characteristic excitation and emission wavelengths for $\operatorname{AMN}\left(\lambda_{\mathrm{ex}}=405 \mathrm{~nm}\right.$ and $\left.\lambda_{\mathrm{em}}=550 \mathrm{~nm}\right)$.

\section{In vitro Exposure of McA-RH7777 Hepatoma Cells to Amonafide Loaded Magnetic Micro- spheres}

A McA-RH7777 hepatoma cell line (ATCC, CRL-1601, Manassas, VA, USA) was cultured in Dulbecco's Modified Eagle's Medium (DMEM, ATCC, Manassas, VA, USA) supplemented with $10 \%$ fetal bovine serum (Sigma-Aldrich, MO, USA) and 0.1\% gentamycin (Sigma-Aldrich, MO, USA). Cells from 
the exponential phase of the culture were harvested and diluted to a cell density of about $2 \times 10^{4}$ per ml. 100 $\mathrm{ul}$ of the cell suspension was added to $180 \mathrm{ul}$ of medium in each well of a 96-well plate, incubated at 37 ${ }^{\circ} \mathrm{C}, 5 \% \mathrm{CO}_{2}$ and $95 \%$ air for 1 day. A 100 ul solution consisting of different amounts of either free AMN or AMN-magnetic microspheres $(20 \mathrm{wt} \%$ magnetic clusters) was then added to the respective wells and incubated for specific periods of time. Studied concentrations of AMN (within either free AMN or AMN-loaded magnetic microsphere doses) ranged from 1.25 to $50 \mathrm{uM}$. Control studies were conducted using dose wells with normal saline. Exposure time was $40 \mathrm{~h}$. Treated cells were then rinsed with PBS before $20 \mathrm{ul}$ of PBS containing $5 \mathrm{mg} / \mathrm{ml}$ of MTT was added prior to incubation for another $4 \mathrm{~h}$. This was followed by the addition of $150 \mathrm{ul}$ of DMSO and plate agitation for $10 \mathrm{~min}$. The optical density (OD) of the contents in each well was then measured at $570 \mathrm{~nm}$ using a bioassay reader (SpectraMax M5, Molecular Devices, CA, USA). OD measurements were repeated in triplicate. Cell viability for each sample was calculated as the ratio between OD measurements within control and treatment wells $\left(\%\right.$ viability $=\left(\mathrm{OD}_{\text {treatment }} /\right.$ $\left.\mathrm{OD}_{\text {control }}{ }^{*} 100\right)$. Significant differences were determined using the Student's t-test where differences were considered significant $(p<0.05)$. For additional cell death analyses, McA-RH7777 cells were treated with AMN-loaded magnetic microspheres (AMN: 25 $\mathrm{uM}$ ) for increasing exposure periods of 4,8 and $12 \mathrm{~h}$ prior to an additional unexposed incubation period for $12 \mathrm{~h}$. Next, for each of these samples FACS analysis (Becton Dickinson LSR Fortessa flow cytometer) was used to determine the percentage of necrotic cells (propidium iodide positive, annexin $\mathrm{V}$ positive), and early apoptotic cells (propidium iodide negative, annexin $V$ positive). Data were collected using CellQuest software (BD Biosciences) and analyzed using FCS Express (De Novo Software).

\section{Characterization of MR Relaxivity Properties}

$\mathrm{T} 2$ and $\mathrm{T} 2 *$ relaxation times for the magnetic clusters and magnetic microspheres were determined using a 7 Tesla MRI scanner (BioSpec, Bruker, Billerica, MA, USA). Imaging phantoms were prepared by diluting samples in $1 \%$ agarose at various different particle or microsphere concentrations. For the magnetic clusters, the atomic Fe concentrations of the stock solutions were determined using Inductively Coupled Plasma Spectroscopy (Inductively Coupled Plasma Mass Spectrometer (ICP- MS), Perkin Elmer, Waltham, MA, USA) and MRI signal changes were measured with increasing $\mathrm{Fe}$ concentrations. For magnetic microspheres, samples were suspended within agarose phantoms (magnetic microspheres concentrations of $0,0.25,0.5,1.5,2.5$, and $3.5 \mathrm{mg} / \mathrm{mL}$ ). For T2 measurement, a Carr-Purcell-Meiboom-Gill (CPMG) sequence of 6 echos was used with $\mathrm{TR}=1000 \mathrm{~ms}$ and $\mathrm{TE}=6.4 \sim 44.8 \mathrm{~ms}$ with an echo interval of $6.4 \mathrm{~ms}$. For the $\mathrm{T}^{*}$ measurements, a multi-echo gradient echo sequence was used with $\mathrm{TR}=$ $700 \mathrm{~ms}, \mathrm{TE}=6.4 \sim 44.8 \mathrm{~ms}$ with interval of $6.4 \mathrm{~ms}$. The $\mathrm{T} 2$, and $\mathrm{T} 2 *$, were calculated using a least squares single exponential fitting model in pixel by pixel and then averaging over the ROIs. For each concentration, we performed a linear fit between relaxation value and particle concentration with corresponding slope thus providing relaxivity estimate (Origin 7.0, Northhampton, MA).

\section{McA-RH7777 Rat Hepatoma Model}

Animal studies were performed with approval from Institutional Animal Care and Use Committee (IACUC). McA-RH7777 hepatoma cells were implanted in the left lateral liver lobe during mini-laparotomy procedures in 10 male Sprague Dawley rats (Fig. 6a). Briefly, rats were anesthetized with isoflurane (mixture of $5 \%$ isoflurane and oxygen at $3 \mathrm{~L} / \mathrm{min}$ ). The sedation was maintained with dose of $1-2 \%$ isoflurane and oxygen flow rate of $3 \mathrm{~L} / \mathrm{min}$. After shaving and sterilization, a mini-laparotomy performed and left hepatic lobe exposed on sterile compress. $1 \times 10^{6}$ McA-RH7777 cells were injected in the left lateral lobe and surgical site closed in 2-layers. Topical antibiotic ointment was applied followed by Metacam injection $(1 \mathrm{mg} / \mathrm{kg}, \mathrm{SQ})$. Tumors were allowed to grow for 7 days to reach a size typically $>5 \mathrm{~mm}$ in diameter while observing animal daily for any signs of distress.

\section{Hepatic Intra-arterial Transcatheter Infusion of Amonafide Loaded Magnetic Microspheres}

After 7 days of tumor growth, the following steps were used to invasively catheterize the left hepatic artery (LHA) for selective infusion of the magnetic microspheres in each animal. First, rats were anesthetized with an isoflurane induction dose (mixture of $5 \%$ isoflurane and oxygen at $3 \mathrm{~L} / \mathrm{min}$ ). After laparotomy, a cotton-tipped applicator was used to expose the common hepatic artery (CHA), proper hepatic artery (PHA), and gastroduodenal artery (GDA) (Fig. 6b). A micro bulldog clamp was placed on the CHA (World Precision Instruments, Sarasota, FL) to prevent bleeding during catheterization. 4-0 Vetacryl absorbable polyglycolic acid suture (Webster Veterinary, Devens, MA) was then used to ligate the GDA distally to control retrograde bleeding during catheterization. Next, a 24G SureFlash polyurethane catheter (Terumo Medical Co., Somerset, NJ) was inserted into the GDA, advanced into the PHA and then 
distally into the LHA. X-ray digital subtraction angiographic (DSA) was used to confirm catheter placement in common branch of PHA using iodinated contrast (Omnipaque, Amersham) (Fig. 6c). After selective catheterization, $0.1 \mathrm{~mL}$ of heparin was infused before infusing the magnetic microspheres $(5 \mathrm{mg}$ in $200 \mathrm{ul}$ ); each infusion was followed by a $0.2 \mathrm{~mL}$ saline flush. The catheter was then withdrawn, and a 3-0 suture used to permanently ligate the GDA above the insertion position. Finally, abdomen was closed using two-layer technique. The animals were then moved to the MRI scanner located adjacent to the surgical suite.

\section{MRI Visualization of Microsphere Delivery}

T2*-weighted images were collected pre- and post- arterial infusion of the magnetic microspheres (as described above). MR scans were performed in coronal orientation using a gradient-echo sequence with following parameters: TR/TE $=1,300 / 7.2 \mathrm{~ms}$, $0.7 \mathrm{~mm}$ slice thickness, FOV $71 \times 85 \mathrm{~mm}, 216 \times 256$ matrix, respiratory triggering with MRI-compatible small animal gating system (Model 1025, SA Instruments, Stony Brook, NY).

\section{Histology}

Each rat was euthanized after catheterization and imaging procedures. HCC specimens were sliced at $2 \mathrm{~mm}$ intervals; these slices were sectioned into 5 um thick sections for hematoxylin and eosin (H\&E) and Prussian blue staining to identify regions of HCC tissue and magnetic microspheres delivery to the tumors. All slides were digitized at x200 optical magnification using a TissueFAXS microscope (TissueGnostics $\mathrm{GmbH}$, Vienna, Austria). Post-processing was performed using the HistoQuest software package (TissueGnostics $\mathrm{GmbH}$ ).

\section{Results}

\section{Microfluidic Fabrication of MRI Visible Drug Eluting Microspheres}

We synthesized $\sim 42 \mathrm{~nm}$ magnetic clusters (Fig. 1b). Each magnetic cluster is a supramolecular structure formed by controlled agglomeration of USPIO nanoparticles. The magnetic cluster solution was strongly water-dispersible and stable in aqueous solution with a surface charge of $-48.7 \mathrm{mV}$ (zeta-potential). $82 \%$ of initial amount of AMN was loaded onto the magnetic clusters. These AMN-magnetic clusters were incorporated into the microsphere drug delivery platform using a microfluidic channel (Fig. 1c). These microfluidic fabrication methods produced 34 um-magnetic microspheres when using $5 \mathrm{ul} / \mathrm{min}$ and $20 \mathrm{ul} / \mathrm{min}$ flow rates for the dispersed and continuous oil phase solutions, respectively. The sheared microsphere aqueous phase in the

microfluidic channel was gelled into solid magnetic microspheres upon contact with $\mathrm{CaCl}_{2}$ solution by crosslinking (Fig. 1c). The AMN-magnetic clusters were well encapsulated in the microspheres, visualized as punctate white spots within monochrome confocal microscopy images (Fig. 1d and e). The shape of the microspheres remained spheroidal after the crosslinking gelation (Fig. 1d and e). Approximately $20 \mathrm{~nm}$ pores on the surface of the dehydrated magnetic microspheres were measured (Fig. 1f (see inset)).

\section{Microsphere Size Distribution and Magnetic Properties}

The measured average diameter of the microspheres was 34 um with a variation of $8 \%$, as demonstrated by the size distribution of these magnetic microspheres in aqueous solution (Fig. 2a). These microspheres demonstrated superparamagnetic properties preserving the magnetic properties of the magnetic clusters (Fig. 2b).

(a)
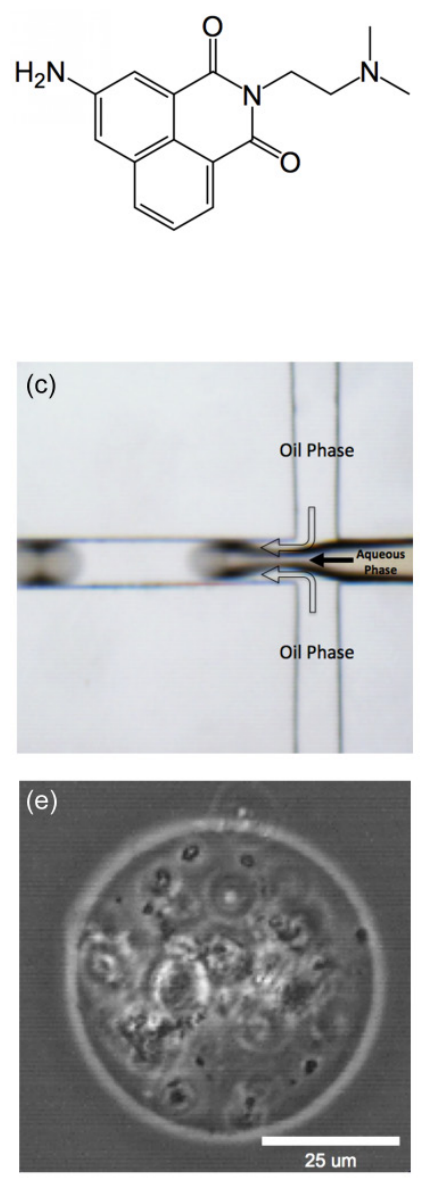

Fig. 1. (a) Chemical structure of amonafide, (b) TEM image of the magnetic clusters, (c) optical microscopy image of flow-focusing channel in microfluidic device (channel width $=130 \mathrm{um})$, ( $\mathrm{d}$ and e) confocal microscope monochrome images and (f) SEM image of microfluidics synthesized magnetic microspheres with magnified inset (dashed box) depicting positions of representative pores (arrows). 

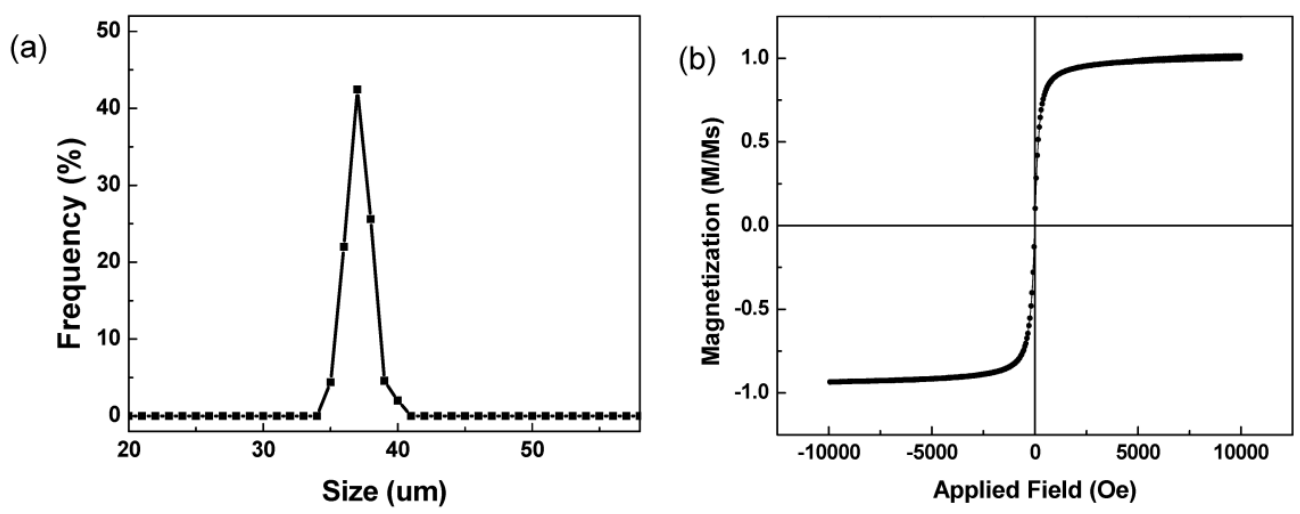

Fig. 2. (a) Size distribution and (b) magnetic properties of magnetic microspheres synthesized with microfluidic system.

\section{AMN Loading and Release}

AMN with magnetic clusters was effectively loaded into the magnetic microspheres; AMN loading efficiency was $55 \%$ relative to the feeding constituent. Loaded AMN was confirmed with fluorescent images that showed the AMN distribution within the microspheres. AMN was dispersed throughout the microspheres (Fig. 3b). Drug-loaded magnetic microspheres (those co-encapsulating magnetic clusters) demonstrated significantly slower AMN release rates compared to those microspheres encapsulating only the drug (Fig. 3c). Only 47\% of the cumulative dose drug dose was released within $24 \mathrm{hrs}$ from the magnetic microspheres whereas $90 \%$ of the cumulative dose was released over the same time period from the alginate-only microspheres without magnetic clusters.

\section{McA-RH7777 Hepatoma Cell Growth Inhibi- tion}

Over an exposure period of 40 hours, equivalent increasing doses of both free AMN and AMN-magnetic microspheres (from 1.25 to $50 \mathrm{uM}$ AMN concentrations) led to corresponding dose dependent decreases in McA-RH7777 hepatoma cell viability (Fig 4a). At lower microsphere dose levels, McA-RH7777 cell viability was much higher in samples exposed to AMN eluting magnetic microspheres when compared to for samples treated with equivalent doses of free-AMN. The viabilities of cells treated with AMN-magnetic microspheres were significantly higher than free AMN treated cells $(\mathrm{p}<0.05)$. The differences $(\sim 45 \%)$ of viabilities at $1.25 \sim 12.5 \mathrm{uM}$ of AMN were decreased to 5 10\% with increased amounts of AMN $(25$ and $50 \mathrm{uM})$ in free AMN and AMN-magnetic microspheres. Extending the exposure period to AMN eluting microspheres $(25 \mathrm{uM})(4$, 8 and 12 hour periods) increased cell death and apoptosis (Fig. $\mathbf{4 b}$ ).

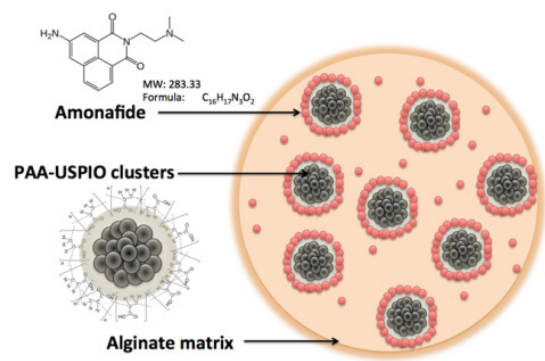

(b)

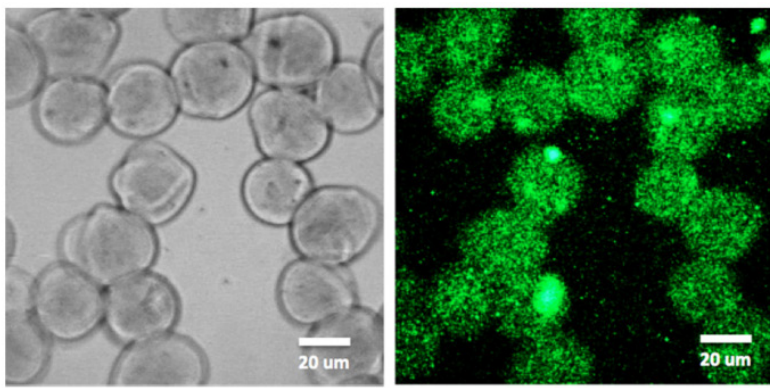

(c)

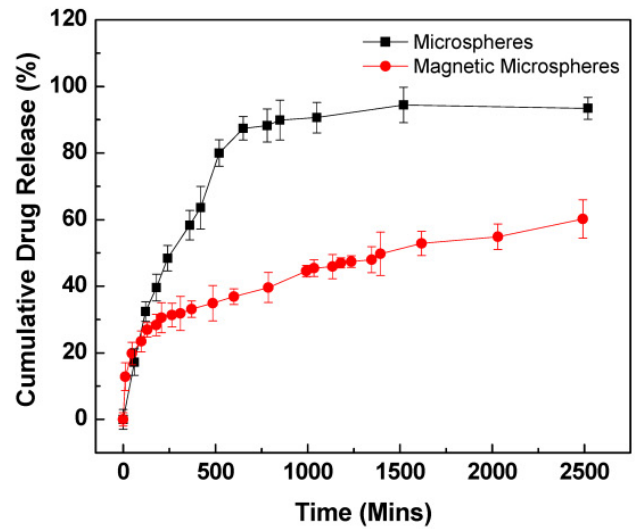

Fig. 3. (a) Schematic illustration of $A M N$-magnetic microspheres, (b) Confocal fluorescent microscope monochrome (left) and fluorescent (right) images of AMN loaded magnetic microspheres $\left(\lambda_{e x}=405\right.$ and $\left.\lambda_{e m}=585 \mathrm{~nm}\right)$ and (c) drug release of $\mathrm{AMN}$ from alginate microspheres and magnetic alginate microspheres including $20 \mathrm{wt} \%$ AMN-magnetic clusters. 
(a)

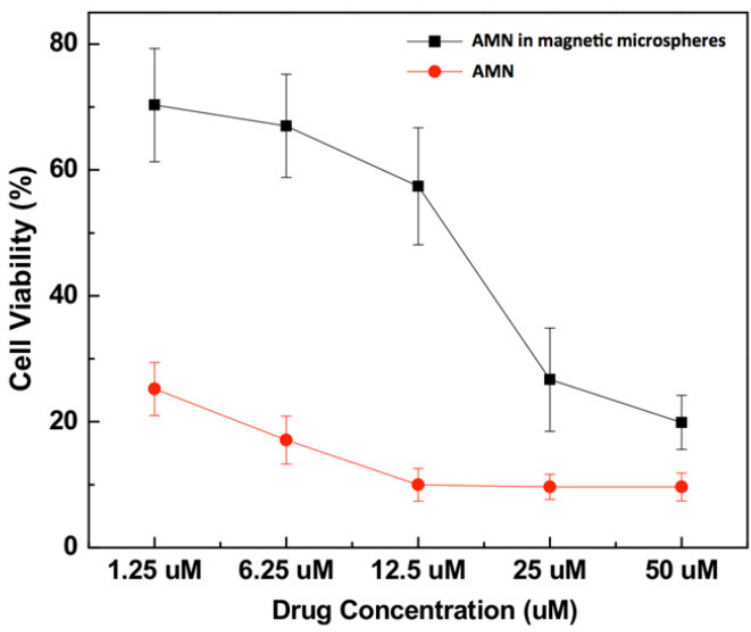

(b)
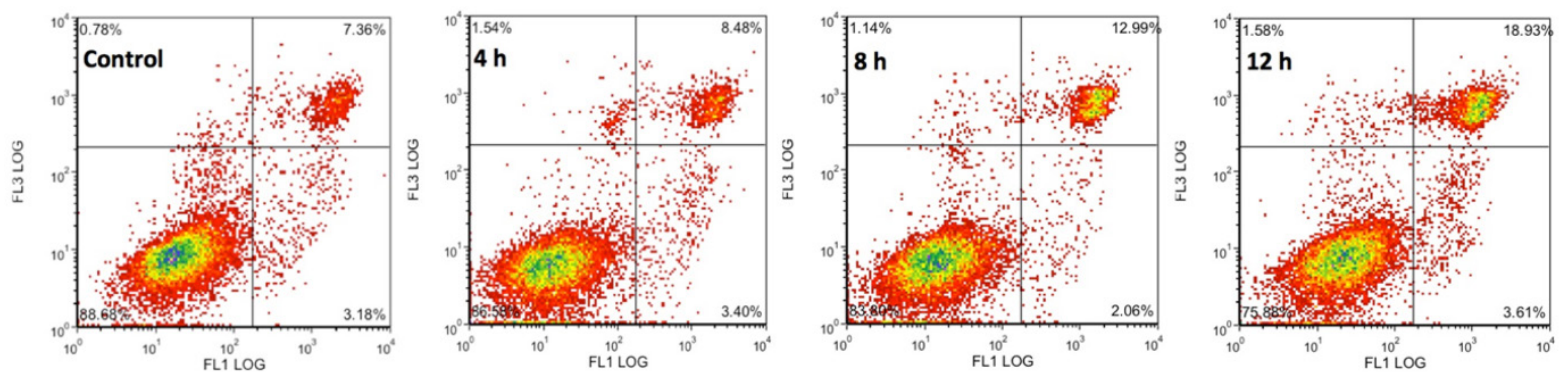

Fig. 4. (a) Dose dependent reductions in McA-RH7777 cell viability upon exposure to free AMN and AMN loaded magnetic microspheres (including 20 wt\% AMN-magnetic clusters) for $40 \mathrm{hr}$ period and (b) impact of AMN eluting magnetic microspheres (25 uM dose) upon McA-RH7777 hepatoma cells after increasing exposure periods $(4,8$ and $12 \mathrm{~h})$.

(a)
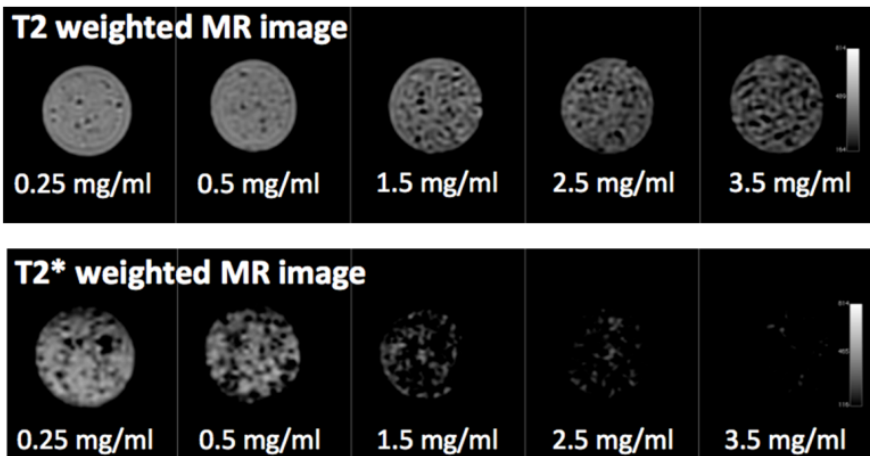

(b)

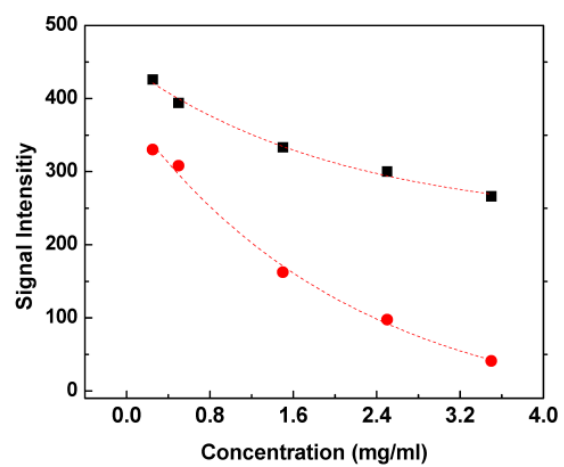

Fig. 5. (a) $M R T 2$ and $T 2 *$ weighted images at $T E=32 \mathrm{~ms}$ showing the expected contrast effects upon phantom signal intensity with increasing concentration of the magnetic microspheres and (b) plots of T2 (square dots) and T2* (circle dots) signal intensity versus magnetic microsphere concentrations in $1 \%$ agar phantoms $\left(r 2=1.32 \mathrm{mg}^{-1} \mathrm{~s}^{-1}\right.$ and r $2 *=6.06$ $\left.\mathrm{mg}^{-1} \mathrm{~s}^{-1}\right)$.

\section{MR Imaging Characteristics of Magnetic AMN Eluting Microspheres}

Agar phantom signal intensities during MR imaging decreased with increasing concentration of magnetic microspheres encapsulating $20 \mathrm{wt} \%$ magnetic clusters (magnetic cluster $\mathrm{r} 2=302 \mathrm{mM}^{-1} \mathrm{~s}^{-1}$ ) (Fig. 5a). Across the five magnetic microspheres concentrations evaluated, T2 and T2* weighted signals decreased exponentially in proportion to increasing microsphere concentration (Fig. 5b); calculated R2 and R2* relaxivities from these measurements were $1.32 \mathrm{mg}^{-1} \mathrm{~s}^{-1}$ and 6.06 $\mathrm{mg}^{-1} \mathrm{~s}^{-1}$, respectively.

\section{Catheterization and MRI Visualization of Magnetic Microsphere Delivery}

Tumor growth following implantation of McA-Rh7777 cells was confirmed in each rat prior to catheterization. Tumor sizes ranged from 2.5 to $8.1 \mathrm{~mm}$ in diameter 7 days post-implantation. Catheterization procedures were successfully performed in each animal (Fig. 6). DSA images were used to confirm position of catheter prior to microsphere arterial infusion. Within MRI images, tumors were 
depicted as hyperintense relative to surrounding liver tissues in T2*-weighted images (Fig. 6c). Representative $\mathrm{T} 2{ }^{*}$-weighted images acquired before and immediately after transcatheter magnetic microsphere infusion is shown in Fig. 6c. Intra-hepatic microsphere delivery was restricted to the targeted tumor-bearing liver lobe with positions of microsphere deposition observed as punctate local reductions to the signal intensity within the

Fig. 6. (a) Catheterization of the hepatic artery proper, intraluminal catheter placement without vessel puncture indicated by flashback of blood into the catheter and (b) (left) coronal and (right) magnified (yellow circle region) images during hepatic $x$-ray angiography at the proper hepatic artery before infusion of the AMN-magnetic microspheres and (c) in vivo transversal $\mathrm{T} 2 *$-weighted $\mathrm{MR}$ images (TR/TE: $1300 \mathrm{~ms} / 7.2 \mathrm{~ms}$ ) of tumor regions within left hepatic lobe acquired pre and post-transcatheter infusion of AMN-magnetic microspheres in McA-RH7777 rat HCC models. Intra-hepatic microsphere deposition was depicted as punctate regions (arrows) of signal loss (insets: magnified tumor regions)

(a)

(c)
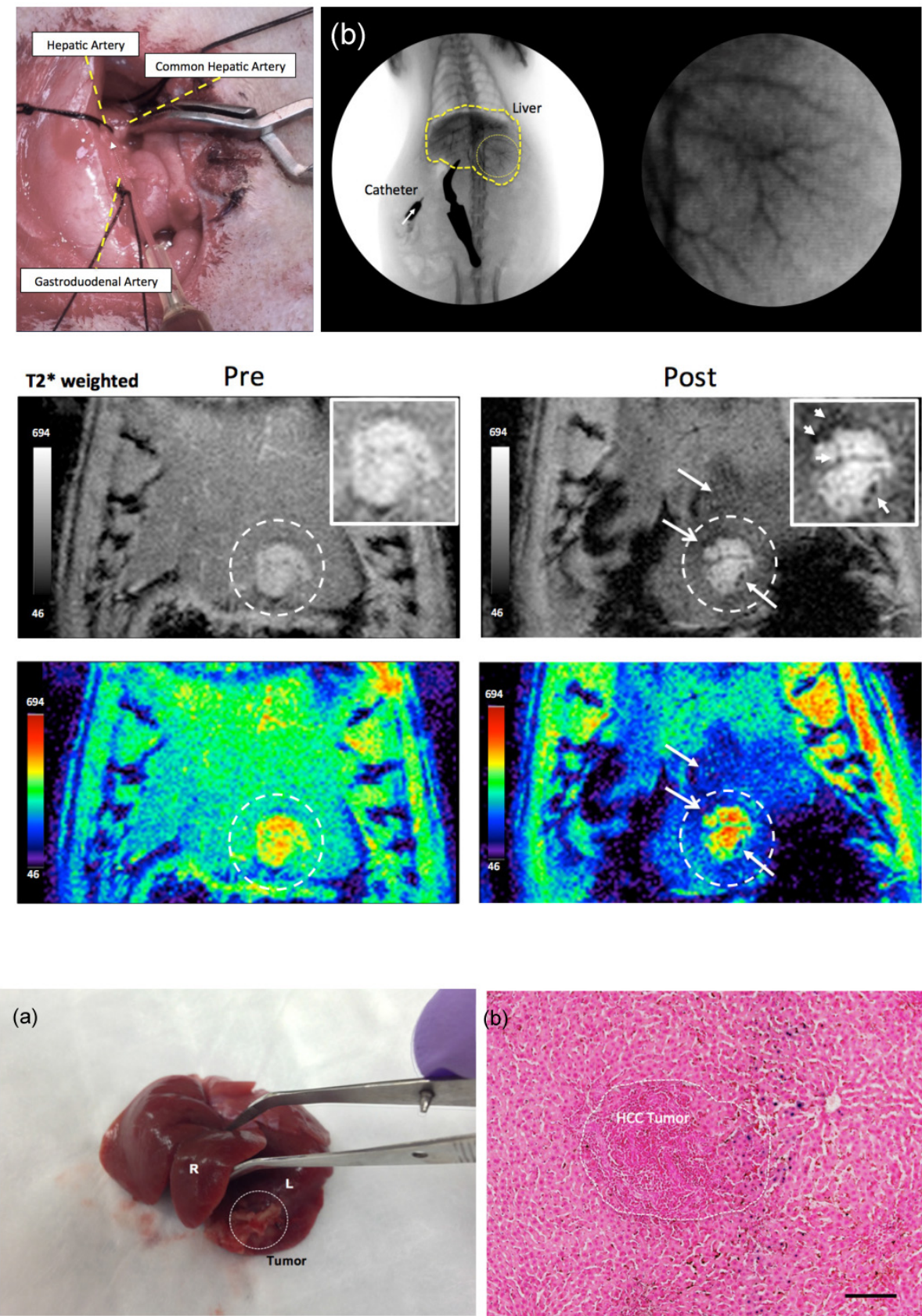

Fig. 7. Optical Images of (a) extracted liver showing tumor region (dashed circle, L:left lobe, R:right lobe), (b) Prussian blue stained liver tumor tissues in a treated McA-RH7777 rat hepatoma (scale bar $=100 \mathrm{um}$ ), (c) magnified regions of magnetic microsphers in vessels (scale bars $=50 \mathrm{um}$ ). Prussian blue staining of treated tumor tissues confirmed selective delivery of the magnetic microspheres to both tumor and the normal liver segment containing that tumor (dark blue magnetic microspheres deposits well depicted from representative histology slide).
T2*-weighted images (Fig. 6c). Each liver was extracted and successful microsphere delivery to both tumor and the normal liver segment containing that tumor was confirmed with Prussian blue staining in rat tissue specimens post-necropsy (Fig. 7). 


\section{Discussion}

TACE has been a mainstay for the treatment of patients with intermediate-stage primary liver cancer.[19] TACE results in high intra-tumoral concentration of chemotherapy drugs. Conventional TACE methods involve the use of lipiodol (oil) mixed with chemotherapy drugs (such as doxorubicin, mitomycin, cisplatin or drug mixtures). The lipiodol helps to increase the contact time between chemotherapy drugs and tumor cells due to temporary embolization of the arteries thus slowing wash-out rates (typically embolic materials also infused to additional blockage of blood flow). However, recent data suggests that the systemic release of chemotherapy agents following conventional TACE is high, and many patients still suffer side effects.[20-22] A recent advancement in the treatment of HCC involves the use of drug-eluting microspheres, which advantageously combine functions of imaging visibility, transcatheter arterial administration for tumor targeting and controlled release of chemotherapeutic drugs. Careful selection of drug carrier materials, chemotherapeutic drugs and fabrication methods will be critical for the translational optimization these new techniques. In our study, magnetic clusters, the drug AMN and alginate polymers were successfully combined into a tunable and uniform microsphere structure fabricated using microfluidic techniques. The potential utilization of these synthesized AMN eluting magnetic microspheres for transcatheter targeted HCC treatment was demonstrated with tests of in vitro biocompatibility and hepatoma cell growth inhibition. Further in vivo hepatic arterial infusions were performed to demonstrate the feasibility of imaging the intra-arterial delivery of these AMN eluting magnetic microspheres in an orthotropic rodent HCC model.

Magnetic clusters are known to provide superior MRI contrast than conventional USPIO (less than $10 \mathrm{~nm}) \cdot[23,24]$ The synthesized magnetic clusters in current study demonstrated 3 times higher $\mathrm{r}_{2}$ relaxivity than non-clustered $7 \mathrm{~nm}$ USPIO nanoparticles.[18, 25] These strong MR contrast effects should be valuable when incorporating the magnetic clusters within drug delivery vehicles for MRI-guided procedures. FDA approved biodegradable polysaccharide alginate polymers were used for the microsphere matrix. Alginate importantly offers unique properties including biocompatibility, a relatively inert environment within the matrix, and a mild room temperature gelation process. To date, the production of alginate microspheres has primarily been accomplished using dropping, electrostatic dripping, extrusion, and/or emulsification methods. Recently developed microfluidic offer to generate more uniform microspheres and the possibly to integrate additional functionalities with the inclusion of proteins, cells, multi-drug cocktails, and imaging probes.[26-31] The ionic crosslinking property of the sodium alginate with calcium ions facilitates in situ microfluidic fabrication of mono-dispersed alginate microspheres. Properties such as microfluidic channel geometry, flow rate, and viscosity can be readily adjusted to control resulting microsphere characteristics. In our microfluidic setup, symmetric shearing of the continuous phase (alginate and magnetic clusters) by the dispersed oil phase $\left(\mathrm{n}^{\prime}\right.$-hexadecane and span80) at a controlled flow rate enabled stable adjustments to the size of the resulting microspheres. 25 60 um magnetic microspheres were fabricated with flow rate ranges of dispersed oil phase $(3 \sim 40 \mathrm{uL} / \mathrm{min})$ and continuous aqueous phase ( 2 and $5 \mathrm{uL} / \mathrm{min}$ ) (Supplementary Material: Fig. S1). At each flow-rate condition, uniform sized magnetic microspheres were produced with size distributions within 5 10\%.

AMN chemotherapeutic drug act a highly effective DNA intercalator; while potentially efficacious against advanced staged tumors[32, 33], AMN has unacceptable side-effects following systemic administration limiting patient tolerance and complicating the dosing of this drug.[34, 35] Therefore, functional drug carriers that control drug release after targeted tumor regional delivery will be required for AMN to serve as an effective viable option for the treatment of liver tumors. In our study, AMN was loaded into 34 um-magnetic microspheres. AMN drug release kinetics from the alginate magnetic microspheres were measured and compared with alginate-only microspheres AMN was released in a time dependent fashion with two phases of release including a rapid initial release phase (first order release) followed by a slower sustained release (zero-order release) period (Fig. 3c). The fast drug release during initial 2 hours was likely due to loosely bound AMN-magnetic clusters at the microsphere surface. When $20 \mathrm{wt} \%$ magnetic clusters were incorporated within the alginate microspheres, the loading efficiency was 55\%, much higher than the drug loading efficiency achieved without inclusion of the magnetic clusters (24\%). Furthermore, the AMN-magnetic clusters loaded microspheres demonstrated sustained drug release characteristics ( $47 \%$ cumulative drug release within one day and lasted for 2 weeks) whereas drug release rates were much more rapid from alginate-only microspheres $(90 \%$ cumulative drug release within one day). These results indicate that magnetic clusters have the potential to control drug release rates. The PAA anionic surface on the magnetic clusters likely contributed to the high drug loading efficiency and sustained drug release. Many of the side 
chains of PAA on the magnetic clusters lose their protons and acquire a negative charge. This makes PAAs polyelectrolytes, with the ability to absorb with electrostatic bonding and retain aqueous solution including AMN drugs having amine groups (Fig. 3a). Addition, the magnetic clusters entrapped in the alginate matrix may block paths of drug diffusion slowing release rates. The benefits of sustainable release and precise dose control should be considerable offering to enhance anti-cancer effects during image-guided HCC therapies.

These sustained drug release characteristics were functionally during our in vitro cell studies. Upon HCC cell exposure to AMN loaded magnetic microspheres, initial viabilities remained higher than for cell samples exposed to equivalent amounts of free AMN drug (less initial exposure to the drug). Additionally, FACS analysis clearly demonstrated a time-dependence for resulting necrosis and apoptotic cell death levels induced by AMN released from the magnetic microspheres. Local delivery and sustained drug release characteristics should be particularly advantageous for AMN offering the potential to reduce serious side effects that may occur with initial high concentrations. Importantly, these studies also demonstrated that $\mathrm{AMN}$, initially encapsulated and then eluted from these magnetic alginate microspheres, maintained potency (cell killing ability) over a relatively long time period.

The functional magnetic clusters contained within the microspheres played an additional role permitting MRI detection given strong T2 and T2* relaxivity effects. As shown in Fig. 5a, the T2 and T2* contrast effects of magnetic microspheres were confirmed with heterogeneous signal reductions caused by some level of agglomeration of the magnetic microsphere during the phantom preparation process. With increasing concentrations of these magnetic microspheres, T2 and T2* weighted MR signal was significantly decreased thus allowing in vivo detection following transcatheter delivery to liver tumors our Sprague Dawley McA-RH7777 xenograft rat model. MRI visibility could be highly advantageous in clinical setting permitting either procedural adjustments (arterial catheter repositioning to better target the tumors) or early prediction of treatment outcomes and identification of treatment failure. Of particular practical importance, the prepared magnetic microspheres $(\sim 20 \mathrm{mg} / \mathrm{ml})$ reached a stable suspension not exhibiting any significant precipitation at the time of intra-arterial infusion. These were able to be infused through the catheter with minimal application of pressure. MR imaging and Prussian blue staining of treated tumor tissues confirmed selective delivery of the magnetic microspheres to both tumor and the normal liver segment containing that tumor. For the distribution of magnetic microspheres, imaging and subsequent necropsy for histology sample preparation actually took place well before the microspheres would be broken down for release of the encapsulated iron-oxide materials and subsequent RES uptake (and of course the microspheres themselves, prior to degradation, would remain intra-vascular given diameters ranging from 17 to $40 \mathrm{um}$.[36]) Delaying imaging for extended period post-infusion could indeed lead to RES uptake of release iron-oxide nanoparticles in such a way that may not accurately reflect the initial microsphere distribution immediately post-infusion. This potential limitation may be critical to consider in future longitudinal pre-clinical and/or clinical translational studies.

Cytotoxic effect of chemotherapy in regional tumor was positively correlated with the regional drug concentration. Thus, the best treatment model for cancer is to increase chemotherapeutic drug concentration in regional tumor, at the same time decreasing drug concentration in peripheral blood as possible to reduce systemic toxicity. In our study, the microfluidic fabricated 34 um-magnetic microspheres will deliver AMN drug locally by hepatic artery infusion permitting a release of drugs into the tumor blood supply. The advantage of this technique is higher chemotherapy concentration in the tumor over intravenous systemic chemotherapy. When drug loaded microspheres are infused in the selective hepatic artery, higher concentration remains in the tumor for a long time. AMN loaded in the magnetic microspheres decrease the washout of chemotherapy by the blood stream, which is beneficial to prolong the contact time of chemotherapy with tumor cell and increase uptake of chemotherapy by tumor cells.[37] In vivo pharmacokinetics and therapeutic effects to determine best concentration of AMN should be studied for HCC in future.

\section{Conclusions}

Monodisperse MR visible magnetic microspheres were synthesized using a high-throughput microfluidic procedure. Microfluidic methods effectively produced hydrophilic, deformable, and non-aggregated biocompatible magnetic alginate microspheres with a small size distribution. MR visibility of the magnetic microspheres was demonstrated with strong T2 and T2*-weighted contrast. The magnetic clusters embedded into the alginate matrix controlled drug release rates and prevented initial burst drug release, resulting in sustained release. The synthesized magnetic microspheres were successfully administered via hepatic arterial routes; MRI permitted in vivo imaging of microsphere delivery to both 
tumor and the normal liver segment containing that tumor that was confirmed with histopathology. Further studies that investigate therapeutic responses in rats treated with these AMN loaded magnetic microspheres will be an important next step in the development of this innovative drug delivery system.

\section{Supplementary Material}

Figure S1. http:/ / www.thno.org/v05p0477s1.pdf

\section{Acknowledgements}

This work was supported by Basic Research Grant from ACS (American Cancer Society, ACS 279148) and by four grants R01CA159178, R01CA141047, R21CA173491 and R21EB017986 from the National Cancer Institute and National Institute of Biomedical Imaging and Bioengineering. This work was supported by the Center for Translational Imaging at Northwestern University. Imaging work was performed at the Northwestern University Cell Imaging Facility generously supported by NCI CCSG P30 CA060553 awarded to the Robert H Lurie Comprehensive Cancer Center.

\section{Competing Interests}

The authors have declared that no competing interest exists.

\section{References}

1. Ackerman NB. Experimental studies on the circulation dynamics of intrahepatic tumor blood supply. Cancer. 1972; 29: 435-9.

2. Marelli L, Stigliano R, Triantos C, Senzolo M, Cholongitas E, Davies N, et al. Transarterial therapy for hepatocellular carcinoma: which technique is more effective? A systematic review of cohort and randomized studies. Cardiovascular and interventional radiology. 2007; 30: 6-25. doi:10.1007/s00270-006-0062-3.

3. Biolato M, Marrone G, Racco S, Di Stasi C, Miele L, Gasbarrini G, et al. Transarterial chemoembolization (TACE) for unresectable HCC: a new life begins? European review for medical and pharmacological sciences. 2010; 14: 356-62.

4. Poon RT, Ngan H, Lo CM, Liu CL, Fan ST, Wong J. Transarterial chemoembolization for inoperable hepatocellular carcinoma and postresection intrahepatic recurrence. Journal of surgical oncology. 2000; 73: 109-14.

5. Trinchet JC, Ganne-Carrie N, Beaugrand M. Intra-arterial chemoembolization in patients with hepatocellular carcinoma. Hepato-gastroenterology. 1998; 45 Suppl 3: 1242-7.

6. Song MJ, Chun HJ, Song DS, Kim HY, Yoo SH, Park CH, et al. Comparative study between doxorubicin-eluting beads and conventional transarterial chemoembolization for treatment of hepatocellular carcinoma. J Hepatol. 2012; 57: $1244-50$

7. Gonzalez MV, Tang YQ, Phillips GJ, Lloyd AW, Hall B, Stratford PW, et al. Doxorubicin eluting beads - 2: methods for evaluating drug elution and in-vitro : in-vivo correlation. J Mater Sci-Mater M. 2008; 19: 767-75. doi:Doi 10.1007/S10856-006-0040-Y.

8. Taningher M, Malacarne D, Izzotti A, Ugolini D, Parodi S. Drug metabolism polymorphisms as modulators of cancer susceptibility. Mutat Res-Rev Mutat. 1999; 436: 227-61.

9. Felder TB, Mclean MA, Vestal ML, Lu K, Farquhar D, Legha SS, et al. Pharmacokinetics and Metabolism of the Antitumor Drug Amonafide (Nsc-308847) in Humans. Drug Metab Dispos. 1987; 15: 773-8.

10. Denkbas EB, Seyyal M, Piskin E. 5-fluorouracil loaded chitosan microspheres for chemoembolization. Journal of microencapsulation. 1999; 16: 741-9. doi:10.1080/026520499288681.

11. Liu X, Heng WS, et al. Novel polymeric microspheres containing norcantharidin for chemoembolization. Journal of controlled release: official journal of the Controlled Release Society. 2006; 116: 35-41. doi:10.1016/j.jconrel.2006.08.022.

12. Osuga K, Arai Y, Anai H, Takeuchi Y, Aramaki T, Sugihara E, et al. Phase I/II multicenter study of transarterial chemoembolization with a cisplatin fine powder and porous gelatin particles for unresectable hepatocellular carcinoma: Japan Interventional Radiology in Oncology Study Group Study 0401. Journal of vascular and interventional radiology : JVIR. 2012; 23: 1278-85. doi:10.1016/j.jvir.2012.06.028

13. Morise Z, Sugioka A, Kato R, Fujita J, Hoshimoto S, Kato T. Transarterial chemoembolization with degradable starch microspheres, irinotecan, and mitomycin-C in patients with liver metastases. Journal of gastrointestinal surgery : official journal of the Society for Surgery of the Alimentary Tract. 2006; 10: 249-58. doi:10.1016/j.gassur.2005.08.004.

14. Fu S, Thacker A, Sperger DM, Boni RL, Buckner IS, Velankar S, et al. Relevance of rheological properties of sodium alginate in solution to calcium alginate gel

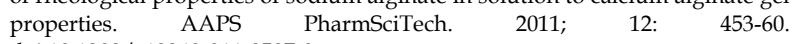
doi:10.1208/s12249-011-9587-0.

15. Thu B, Bruheim P, Espevik T, Smidsrod O, SoonShiong P, SkjakBraek G. Alginate polycation microcapsules .1. Interaction between alginate and polycation. Biomaterials. 1996; 17: 1031-40. doi:Doi 10.1016/0142-9612(96)84680-1.

16. Zuidam NJ, Nedović V. Encapsulation technologies for active food ingredients and food processing. New York ; London: Springer; 2009.

17. Ge JP, Hu YX, Biasini M, Beyermann WP, Yin YD. Superparamagnetic magnetite colloidal nanocrystal clusters. Angew Chem Int Edit. 2007; 46: 4342-5.

18. Kim DH, Guo Y, Zhang Z, Procissi D, Nicolai J, Omary RA, et al. Temperature-sensitive magnetic drug carriers for concurrent gemcitabine chemohyperthermia. Advanced healthcare materials. 2014; 3: 714-24. doi:10.1002/adhm.201300209.

19. Ono $\mathrm{Y}$, Yoshimasu $\mathrm{T}$, Ashikaga $\mathrm{R}$, Inoue $\mathrm{M}$, Shindou $\mathrm{H}$, Fuji $\mathrm{K}$, et al. Long-term results of lipiodol-transcatheter arterial embolization with cisplatin or doxorubicin for unresectable hepatocellular carcinoma. American journal of clinical oncology. 2000; 23: 564-8.

20. Johnson PJ, Kalayci C, Dobbs N, Raby N, Metivier EM, Summers L, et al. Pharmacokinetics and toxicity of intraarterial adriamycin for hepatocellular carcinoma: effect of coadministration of lipiodol. Journal of hepatology. 1991; 13: $120-7$.

21. Lewis AL, Gonzalez MV, Lloyd AW, Hall B, Tang Y, Willis SL, et al. DC bead: in vitro characterization of a drug-delivery device for transarterial chemoembolization. Journal of vascular and interventional radiology : JVIR. 2006; 17: 335-42. doi:10.1097/01.RVI.0000195323.46152.B3.

22. Simonetti RG, Liberati A, Angiolini C, Pagliaro L. Treatment of hepatocellular carcinoma: a systematic review of randomized controlled trials. Annals of oncology : official journal of the European Society for Medical Oncology / ESMO. 1997; 8: 117-36.

23. Mikhaylov G, Mikac U, Magaeva AA, Itin VI, Naiden EP, Psakhye I, et al. Ferri-liposomes as an MRI-visible drug-delivery system for targeting tumours and their microenvironment. Nat Nanotechnol. 2011; 6: 594-602.

24. Paquet C, de Haan HW, Leek DM, Lin HY, Xiang B, Tian GH, et al. Clusters of Superparamagnetic Iron Oxide Nanoparticles Encapsulated in a Hydrogel: A Particle Architecture Generating a Synergistic Enhancement of the T-2 Relaxation. Acs Nano. 2011; 5: 3104-12.

25. Kim DH, Choy T, Huang S, Green RM, Omary RA, Larson AC. Microfluidic fabrication of 6-methoxyethylamino numonafide-eluting magnetic

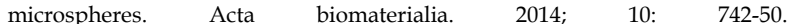
doi:10.1016/j.actbio.2013.10.018

26. Lemoine D, Wauters F, Bouchend'homme S, Preat V. Preparation and characterization of alginate microspheres containing a model antigen. Int J Pharmaceut. 1998; 176: 9-19.

27. Joshi A, Solanki S, Chaudhari R, Bahadur D, Aslam M, Srivastava R. Multifunctional alginate microspheres for biosensing, drug delivery and magnetic resonance imaging. Acta Biomater. 2011; 7: 3955-63.

28. Chen WY, Kim JH, Zhang D, Lee KH, Cangelosi GA, Soelberg SD, et al. Microfluidic one-step synthesis of alginate microspheres immobilized with antibodies. J R Soc Interface. 2013; 10. doi:10.1098/Rsif.2013.0566.

29. Pessi J, Santos HA, Miroshnyk I, et al. Microfluidics-assisted engineering of polymeric microcapsules with high encapsulation efficiency for protein drug delivery. International journal of pharmaceutics. 2014; 472: 82-7. doi:10.1016/j.ijpharm.2014.06.012.

30. Xu Q, Hashimoto M, Dang TT, Hoare T, Kohane DS, Whitesides GM, et al. Preparation of monodisperse biodegradable polymer microparticles using a microfluidic flow-focusing device for controlled drug delivery. Small. 2009; 5: 1575-81. doi:10.1002/smll.200801855.

31. Lagus TP, Edd JF. A review of the theory, methods and recent applications of high-throughput single-cell droplet microfluidics. J Phys D Appl Phys. 2013; 46. doi:10.1088/0022-3727/46/11/114005

32. Liu YN, Norton JT, Witschi MA, Xu Q, Lou GH, Wang C, et al. Methoxyethylamino-numonafide Is an Efficacious and Minimally Toxic Amonafide Derivative in Murine Models of Human Cancer. Neoplasia. 2011; 13: 453-60

33. Allen SL, Lundberg AS. Amonafide: a potential role in treating acute myeloid leukemia. Expert opinion on investigational drugs. 2011; 20: 995-1003. doi:10.1517/13543784.2011.585756.

34. Kornek G, Raderer M, Depisch D, Haider K, Fazeny B, Dittrich C, et al. Amonafide as First-Line Chemotherapy for Metastatic Breast-Cancer. Eur J Cancer. 1994; 30A: 398-400

35. Innocenti F, Iyer L, Ratain MJ. Pharmacogenetics of anticancer agents: Lessons from amonafide and irinotecan. Drug Metab Dispos. 2001; 29: 596-600. 
36. Bastian P, Bartkowski R, Kohler H, Kissel T. Chemo-embolization of experimental liver metastases. Part I: distribution of biodegradable microspheres of different sizes in an animal model for the locoregional therapy. European journal of pharmaceutics and biopharmaceutics : official journal of Arbeitsgemeinschaft fur Pharmazeutische Verfahrenstechnik eV. 1998; 46: 243-54.

37. Zhang YW, Ao J, Liu Y, Qiao MX, Yang XL, Tang SX, et al. Pharmacokinetics of gelatin sponge microparticles in a rabbit VX2 liver tumor model of hepatic arterial chemoembolization. Tumour biology : the journal of the International Society for Oncodevelopmental Biology and Medicine. 2014. doi:10.1007/s13277-014-2408-9. 